\title{
Re: Metastatic pathological fracture of the surgical neck of humerus during manipulation under anaesthesia for adhesive capsulitis
}

\author{
Nicholas A. Ferran · Robert U. Ashford
}

Received: 4 June 2011 / Accepted: 5 June 2011 / Published online: 14 June 2011

(C) Springer-Verlag 2011

We have read the above case report with interest and would like to raise several points with respect to investigation into and management of this case [1].

The patient in this case had a painful shoulder with a history of recurrent breast cancer, and while treatment for breast cancer has been associated with frozen shoulder [2], tumour should have been top of the list of differential diagnoses in this case.

A 4-month history of severe progressive pain, bony tenderness and restricted range of motion also does not fit with the initial phase of adhesive capsulitis in which pain is not usually associated with stiffness and tenderness is usually elicited over the joint capsule [2].

Radiographs are often normal in adhesive capsulitis but may show some decreased bone mineral density [3]; however, in this case, there is a clear lytic lesion in the humeral head and neck on the plain films. It is unclear whether these films were reported by a radiologist. We are of the opinion that an MRI was indicated at this initial stage. An isolated lytic lesion with a distant history ( $>10$ years) warrants further investigation and should be treated as a primary bone tumour until proven otherwise.

The authors claim that conservative management failed to alleviate pain; however, they have not elaborated on the treatment methods used or the duration. By their own admission, capsular release and MUA are used for recalcitrant cases to improve stiffness. Some authors recommend a minimum of 4 months but usually 6 months before considering surgical management [2].

\footnotetext{
N. A. Ferran · R. U. Ashford ( $\square)$

Department of Orthopaedics, University Hospitals of Leicester NHS Trust, Leicester Royal Infirmary, Infirmary Square, Leicester LE1 5WW, UK e-mail: robert.ashford@sky.com
}

It is unclear why the arthroscope was reinserted when a pathological fracture was suspected. Image intensifier films would have provided the diagnosis of fracture without risk of tumour seeding.

A pathological fracture was suspected; however, MRI scan was refused. Biopsy does not seem to have been considered in this case, and its role has not been discussed. A biopsy at this stage may have reduced the 3-month delay in diagnosis. No mention has been made of staging of disease once the diagnosis of metastasis had been made. The tumour was resected and the patient fitted with a spacer. This warrants clarification as the gold standard for treatment of an isolated metastatic proximal humerus metaphyseal fracture is wide excision and replacement with a modular prosthesis [4].

This case as described demonstrates the need for adhesive capsulitis to be a diagnosis of exclusion, highlights the controversies in its management, and illustrates deficiencies in the workup of patients with suspected bone tumours.

Conflict of interest No funds were received in support of this study.

\section{References}

1. Gabr A, Mackay H, Dunn A, Brownson P, Smith MG (2011) Metastatic pathological fracture of the surgical neck of humerus during manipulation under anaesthesia for adhesive capsulitis. Eur J Orthop Surg Traumatol 21:289-292

2. Neviaser AS, Hannafin JA (2010) Adhesive capsulitis: a review of current treatment. Am J Sports Med 38(11):2346-2356

3. Brue S, Valentin A, Forssblad M, Werner S, Mikkelsen C, Cerulli G (2007) Idiopathic adhesive capsulitis of the shoulder: a review. Knee Surg Sports Traumatol Arthrosc 15(8):1048-1054

4. Piccioli A, Maccauro G, Rossi B, Scaramuzzo L, Frenos F, Capanna R (2010) Surgical treatment of pathologic fractures of humerus. Injury 41(11):1112-1116 